DSYNAPTIC REMODELLING

\section{Sculpting the NMJ}

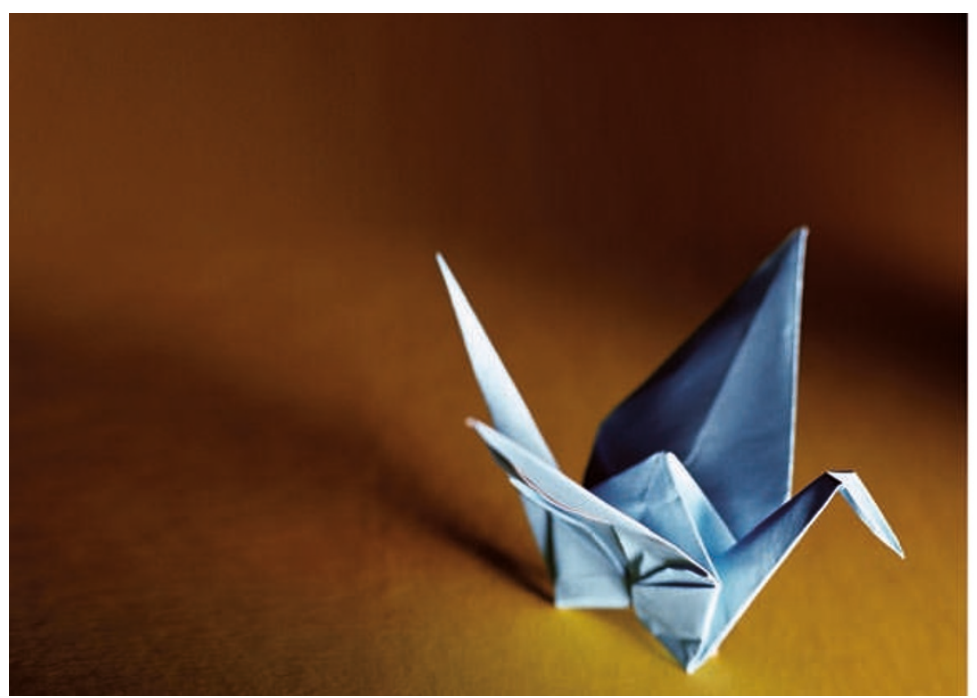

During neuromuscular junction (NMJ) development, target muscle cells become innervated by the axonal arbor of one motor neuron. As the muscle grows, the arbor size increases and new synapses are added. Fuentes-Medel et al. have now examined this process in the Drosophila melanogaster $\mathrm{NMJ}$ in detail and report on a new series of events taking place during synaptic expansion.

The authors examined the NMJ of D. melanogaster larvae and noticed the presence of fragments of debris derived from the presynaptic motor neuron membrane, as well as immature synaptic boutons (known as 'ghost' boutons), in the space surrounding the NMJ. Increasing motor neuron activity - a manipulation that has previously been shown to boost synaptic growth - increased the amount of debris, suggesting that motor neurons shed membrane debris as the NMJ grows and new synapses are added.

The accumulation of presynaptic debris and ghost boutons was not extensive, and the authors therefore postulated that an active clearing mechanism must be at work in the NMJ. Indeed, knocking out draper, which encodes a receptor involved in engulfing neuronal material in other parts of the nervous system, increased the amount of presynaptic debris and the number of ghost boutons. Furthermore, in draper-mutant flies the NMJ developed abnormally, with enlarged or misshapen boutons, suggesting that the clearance of presynaptic material is required for normal NMJ development.

Next the authors studied the cell types responsible for clearing presynaptic debris and ghost boutons. They found that draper was expressed in both peripheral glia and muscle cells at the NMJ, but that each cell type has a distinct role in engulfing the presynaptic material. Knocking down draper function in glia with RNA interference impaired the clearance of presynaptic debris, whereas loss of draper function in muscle cells prevented the clearance of ghost boutons.

The author's findings suggest that, as it grows, the NMJ continuously produces and sheds excess presynaptic membrane material, and that the efficient clearance of this material by glia and muscle cells is vital for normal NMJ development. Whether this process contributes to synapse growth in other parts of the nervous system will be an interesting question for future research.

Katherine Whalley

ORIGINAL RESEARCH PAPER Fuentes-Medel, Y et al. Glia and muscle sculpt neuromuscular arbors by engulfing destabilized synaptic boutons and shed presynaptic debris. PLoS Biol. 25 Aug 2009 (doi:10.1371/journal.pbio.1000184) 